\title{
Application of the Topological Optimization Technique to the Stents Cells Design for Angioplasty
}

S. A. G. Oliveira

Emeritus Member, ABCM sgoulart@mecanica.ufu.br

M. A. Duarte
Senior Member, ABCM
mvduarte@mecanica.ufu.br
Federal University of Uberlândia - UFU
Faculty of Mechanical Engineering
38400-208, Uberlândia, MG. Brazil

\section{Introduction}

The accumulation of substances in the coronary arteries, such as the cholesterol, is one of the causes of death among adults around the world. In the past, most of cardiovascular diseases just were treatable through a by-pass surgery (Serruys and Kutryk, 1998). Nowadays, a minimally invasive catheter-based procedure has been extensively used to unblock the diseased artery. In this procedure, a thin wall metallic tube known as stent is also implanted in the region of the unblocked artery in order to prevent restenosis or re-closure of the blood vessel.

There are several criteria to be considered in the stent design (Serruys and Kutryk, 1998). In this paper, it will be emphasized only the flexibility and the stiffness as the stents mechanical design criteria. During the implant procedure, the stent should be able to track the catheter into the vessel until the stenosed artery region is reached. Thus, the stent should be flexible enough to bend while attached to the catheter. Conversely, the stent should also be sufficiently stiff to maintain the artery opened and to support the pressure applied by the blood vessel wall after the implant.

Analytical prediction of the flexibility and stiffness of a stent only is possible in the linear elastic range (Duerig et al., 2000). Indeed, the structural analysis of a stent is highly nonlinear due to the complexity of its geometry and the large plastic strain that occur during the implant. In this way, the analysis of the design criteria above mentioned has been studied using nonlinear finite elements models or experimental observations of stents implanted in patients (Serruys and Kutryk, 1998; Ahmad and Barrett, 1999; Borgersen and Sadeghi, 2000; Duerig et al., 2000; McClean and Eigler, 2002). On the other hand, the design techniques used in the definition of the stents geometry are not usually described in the literature. It is supposed that the designers use the intuition, experience and the results of the stent analysis to improve their performance (Borgersen and Sadeghi, 2000). However, although these are valuable design tools, they do not guarantee that the obtained stent geometry is optimum. In this context, the structural optimization tools available in the literature provide an automated methodology to obtain the

Paper accepted March, 2008. Technical Editor: Agenor de Toledo Fleury. optimum geometry of stent with maximum flexibility and stiffness (Vanderplaats, 1999).

Topological optimization is a particularly useful design tool to be used in the definition of the best material distribution of a stent (Guimarães, 2005). An advantage of this technique is that problems concerning the maximization of flexibility and stiffness of structures subjected to the large strains are well established in the literature (Yin and Anathasuresh, 2001; Nishiwaki et al., 2001; Mayer et al., 1996; Maute et al., 1998). Then, the same formulation used in the algorithms of topology optimization of stiff and flexible structures can also be applied and adapted to the stents design problem. Therefore, the objective of this work is to employ topology optimization as a mechanical design tool of stainless steel stents by simulating the implant procedure. In the following, it will be described the structural models used in the study of stents, the formulation of the optimization problem and the methodology used in the numerical computation of the stent optimal topology. Finally, the stent optimal material distribution will be shown and compared qualitatively with the geometry of commercial stents.

\section{Nomenclature}

$A=$ factor used in the updating of the flexible topology, dimensionless

$B_{w}=$ factor used in the updating of the hardened topology, dimensionless

$\mathrm{C}_{2} \mathrm{U}_{1}=$ mutual strain energy density, $\mathrm{J} / \mathrm{m}^{3}$

$d V=$ differential element of volume, $\mathrm{m}^{3}$

$E \quad=$ Young's modulus, $N / \mathrm{m}^{2}$

$E_{p} \quad=$ plastic modulus, $\mathrm{N} / \mathrm{m}^{2}$

$E_{t} \quad=$ tangent modulus, $\mathrm{N} / \mathrm{m}^{2}$

$f \quad=$ volume fraction of the optimal topology, dimensionless

$F \quad=$ body force, $N$

$F_{s} \quad=$ surface (normal) force, $N$

$I=$ index used in the summation of the finite elements, dimensionless

$K=$ global stiffness matrix, $N / m$

$m \quad=$ move limit, dimensionless

$N=$ number of finite elements from the design space, dimensionless

$p \quad=$ power of penality of the SIMP model, dimensionless 
$P(x)=$ objetive function used in the maximization of the stent cell flexibility, dimensionless

$U \quad=$ displacement field of the structure, $m$

$V \quad=$ volume of the design space, $m^{3}$

$V(x)=$ volume of the stent cell optimal topology, $\mathrm{m}^{3}$

$W \quad=$ elastic-plastic strain energy density, $\mathrm{J} / \mathrm{m}^{3}$

$x \quad=$ relative density, dimensionless

\section{Greek Symbols}

$\Delta u \quad=$ prescribed displacement applied to the design space of the structure, $\mathrm{m}$

$\varepsilon \quad=$ total strain, dimensionless

$\varepsilon_{e} \quad=$ elastic strain, dimensionless

$\varepsilon_{p} \quad=$ plastic strain, dimensionless

$\eta \quad=$ numerical damping coefficient, dimensionless

$Z \quad=$ load factor, dimensionless

$\sigma_{r} \quad=$ yield stress, $\mathrm{N} / \mathrm{m}^{2}$

$\Gamma \quad=$ region of surface, $\mathrm{m}^{2}$

\section{Subscripts}

$e \quad$ relative to elastic range

$\min$ relative to the minimum value

$o$ relative to properties from the solid material

$p$ relative to plastic range

$r \quad$ relative to yield stress

$t \quad$ relative to tangent

1 relative to force applied by the vessel wall

2 relative to direction of the flexibility

3 relative to reaction force applied by the vessel wall

\section{Superscripts}

e relative to finite element

$j \quad$ relative to iteration index

$T \quad$ relative to transpose

\section{Definition, Characteristics and Models of Stents}

A stent can be defined as any device with circular section used to reinforce the wall of a vessel (Serruys and Kutryk, 1998). As can be seen in Fig. 1, the stent structure is cylindrical and is formed by a repetitive geometrical pattern, known as cells. In applications for angioplasty, the stent diameter before the implant varies of $1.5 \mathrm{~mm}$ to the $2.5 \mathrm{~mm}$. In this phase, the stent should only track the catheter into the vessel. To open arteries where deposits restrict the blood flow, cardiologists use an inflated balloon to push the obstruction aside. When the pressurized balloon inflates, the stent expands, that is, it increases its diameter until it contacts the inner surface of the artery wall (some stents are auto-expansive). After the implant process, the final diameter of the stent implanted in the artery may reach until five times its original diameter (Serruys and Kutryk, 1998; Borgersen and Sadeghi, 2000). The importance of this contact between stent and blood vessel is to prevent any diameter reduction of the artery wall after the implant and also to prevent thrombus formation.

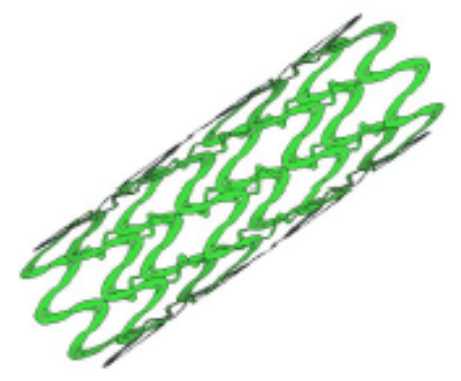

Figure 1. Model of finite elements of a three-dimensional stent (Guimarães et al., 2006).
Figure 2 shows the plane model of a stent with several cells before the expansion process (Guimarães et al., 2006). The expanded stent has some regions where the stress level exceeds the yield stress. The hardening of these regions and the stiffness of the cells maintain the artery wall and the stent with the expanded diameter. In the longitudinal direction of the cell, it can be observed the presence of sinusoidal structures. The role of these structural elements is to improve the flexibility of the stent during the implant, in order to achieve best mobility.

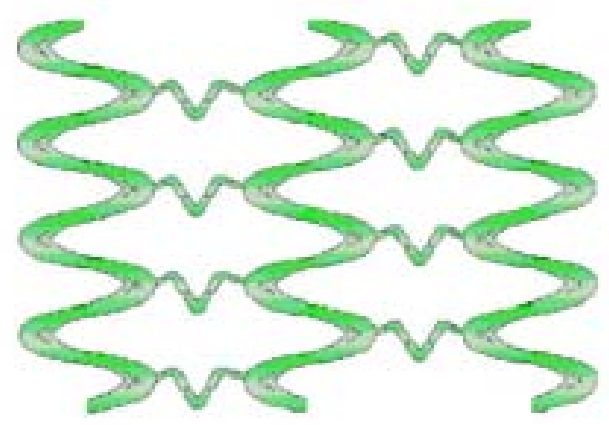

Figure 2. Plane model of the stent with several cells (Guimarães et al., 2006).

\section{Topology Optimization as a Stent Design Tool}

Topology optimization can be defined as a structural design tool that generates automatically the best layout of the elements or components of a structure (Rozvany, 1997). This procedure must locate the structure elements that will be either solid or void. Solid elements have relative density equal to 1 (one) and void elements have relative density equal to 0 (zero). For example, Fig. 3 shows the optimal topology of a cantilever beam with minimal strain energy. In this case, the volume of the optimal topology is $40 \%$ of the original design space volume (rectangular beam).

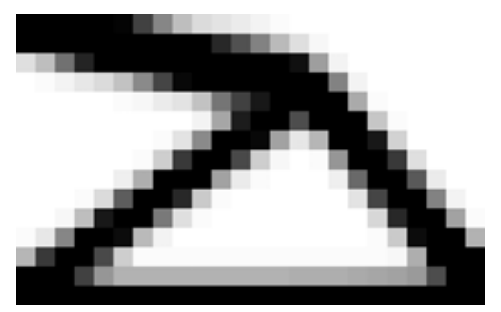

Figure 3. Optimal topology of a cantilever beam.

In this paper, the design problems of the stent cell subjected to the plastic strain provided by the expansion of the balloon and the elastic bending due to the curvature imposed by the catheter will be treated separately using topology optimization. First off all, the topology of the stiff region from the plastically deformed stent cell, shown in the Fig. 2, will be optimized by maximizing the hardening. Subsequently, the elastic strain energy of the flexible longitudinal structure from the stent cell will be maximized and its optimal topology computed. All topologies shown in this paper have a volume equals to $20 \%$ of the total design space volume. This value is recommended and used by the manufacturers in order to avoid the rejection of the stents implanted in the vessel (Serruys and Kutryk, 1999). In fact, the less the metallic surface area of a stent contacts the artery wall, the less is the probability of rejection. 


\section{Stent Cell with Maximum Hardening}

The stent cell should be subjected to plastic strain only during the balloon expansion process. The plastic strain energy absorbed by the stent during the implantation increases the yield strength of the stainless steel. Thus, after the hardening, it will be necessary to increase the stress level in order to expand or compress the diameter of the expanded stent placed in the artery. The purpose of this is to ensure that the stent diameter produced by the angioplasty is maintained.

It is desired to maximize the hardening of the stent to improve the performance during the balloon expansion. One way of maximizing the hardening is to maximize the toughness or total strain energy in the elastic and plastic ranges (Mayer et al., 1996; Maute et al., 1998):

$$
W=\frac{1}{2} \int_{V}\{\varepsilon\}\left[E_{p}\right]\{\varepsilon\} d V
$$

where $d V$ is the volume differential element of a continuous solid body, $\{\varepsilon\}$ represents the total strain defined by the sum of the elastic and plastic strains, $\left\{\varepsilon_{e}\right\}$ and $\left\{\varepsilon_{p}\right\}$, given by (Karasudhi, 1991):

$$
\{\varepsilon\}=\left\{\varepsilon_{e}\right\}+\left\{\varepsilon_{p}\right\}
$$

and $\left[E_{p}\right]$ is the plastic modulus, that is a linear approximation of the stress-strain curve for the elastic and plastic ranges of the stainless steel (Karasudhi, 1991; Mayer et al., 1996). This material model, known as Bilinear Isotropic Hardening (BISO) model, is usually used for large strain analysis of ductile materials, such as a stainless steel stent (Karasudhi, 1991). Another advantage of the BISO model is the fast computation of the stress components of a structure subjected to plastic strain, which is particularly useful in the finite element analysis (Mayer et al., 1996; Ahmad and Barrett, 1999).

The plastic modulus, $E_{p}$, is defined by (Karasudhi, 1991; Mayer et al., 1996):

$$
E_{p}=\frac{E_{t} E}{E-E_{t}}
$$

where $E$ is the Young's modulus and $E_{t}$ is the linear approximation of the plastic range, called tangent modulus. It should be noted that in the Eq. (1), the variable $E_{p}$ denotes the plastic modulus matrix that correlates the equivalent stress tensor with the plastic strain tensor (Mayer et al., 1996). On the other hand, the Eq. (3) is a scalar relation that represents each component of the plastic modulus tensor as a function of the components of the tangent and Young's modulus tensors.

In the BISO model, the definition of the plastic strain increments, $d \varepsilon_{p}$, is based on the flow rule (Karasudhi, 1991; Maute et al., 1998). Geometrically, $d \varepsilon_{p}$ is a normal vector to the yield surface representing the stress state of the structure. For large strain of ductile materials, the Von Mises elliptical yield surface provides the distortion energy caused by any stress combination. Physically, the plastic strain of a ductile material is produced by its distortion energy (Norton, 1996).

Following the topology optimization nomenclature, the mathematical definition of the stent cell design problem with maximum hardening is:

Maximize:

$$
W=\frac{1}{2} \int_{V}\{\varepsilon\}\left[E_{p}\right]\{\varepsilon\} d V
$$

Subjected to:

$$
\begin{aligned}
& f=\frac{V(x)}{V} \\
& \frac{1}{2} \int_{V}\{\varepsilon\}\left[E_{p}\right]\{\varepsilon\} d V=\int_{\Gamma} \zeta\{\Delta u\}^{T}\left\{F_{s}\right\} d \Gamma \\
& 0 \leq x_{\min } \leq x \leq 1
\end{aligned}
$$

such that $f$ is the percentage of the optimal topology volume, $V(x)$, with respect to the total volume, $V$, of the initial design domain. Equation (5) describes the equilibrium of the topology to be satisfied during the optimization. The left side of the Eq. (5) is the total strain energy accumulated by the stent cell. The right one represents the external work of the prescribed displacement, $\{\Delta u\}^{T}$, applied in some region of the surface, $\Gamma$, in the design space. In the surface integral of the Eq. (5), $\left\{F_{s}\right\}$ is an unit and fictitious normal force applied to the design space and $\zeta$, called load factor, represents the magnitude of $\left\{F_{s}\right\}$ that has the same effect of the prescribed displacement, $\{\Delta u\}^{T}$. Equation (6) are the minimum and maximum values (side constraints) that the relative density or design variables, $x$, can assume. The maximum value, $x=1$, is the relative density of the solid material and the minimum value, $x_{\min }=0.001$, of the relative density close to zero is used in order to avoid a singularity of the global stiffness matrix of finite elements.

\section{Stent Cell with Maximum Flexibility}

The stent cell should be flexible as well, since it will be subjected to large deflections in the elastic range when the catheter bends in the blood vessel before the balloon expansion (Serruys and Kutryk, 1998; Borgersen and Sadeghi, 2000). A measure commonly used in the topology optimization field of the structural flexibility, is the mutual strain energy, $C_{2} U_{l}$, defined as follows (Nishiwaki et al, 2001; Yin and Ananthasuresh, 2001):

$$
\mathrm{C}_{2} \mathrm{U}_{1}=\mathrm{U}_{1}^{\mathrm{T}} \mathrm{KU}_{2}
$$

where $U_{l}^{T}$ denotes the displacements of the structure caused by the force $F_{1}, U_{2}$ is the displacement field due to the force $F_{2}$ and $K$ represents the global stiffness matrix of the elastic solid body illustrated in the Fig. 4. Indeed, Eq. (7) measures the displacement or flexibility of the structure in the direction of the unit dummy load, $F_{2}$, when the force $F_{l}$ is applied. Thus, the larger is the mutual strain energy, the larger is the flexibility of the structure.

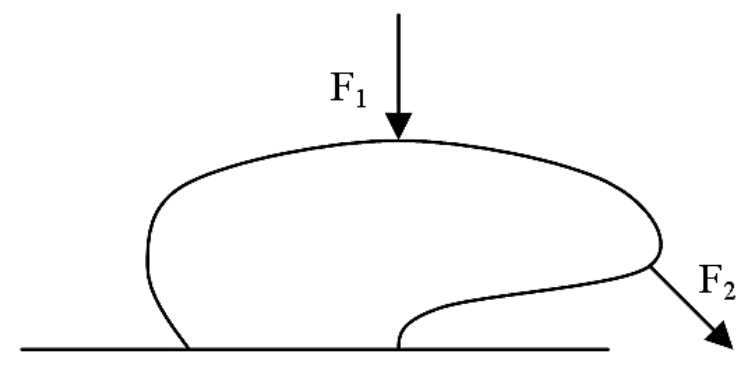

Figure 4. Concept of flexibility of an elastic body.

By considering the flexible stent cell structural model, the force $F_{l}$ may be interpreted as the bending load applied by the blood vessel wall at the same plane of the stent cell. The location of the 
load $F_{2}$ represents the point of contact of the bent stent cell with the vessel wall. Usually, the stent material is stiffer than the vessel wall material (Serruys and Kutryk 1998). Therefore, it is expected that the contact strain is preferentially located in the vessel wall.

Mathematically, the stent cell with maximum flexibility is obtained from the maximization of the Eq. (7). However, the simple maximization of Eq. (7) does not guarantee that the structure will not deform indefinitely. In this case, the problem is ill conditioned and the Eq. (7) will tend to the infinite. The solution for this inconvenient is to limit the mutual strain energy to a finite value during the maximization of the Eq. (7). Most papers dealing with the flexible structures topology optimization introduces a lumped stiffness at the point of application of the forces $F_{1}$ or $F_{2}$ to restrict the flexibility of the structure in the desired direction, (e.g. Nishiwaki et al. 2001; Yin and Ananthasuresh 2001).

In this work, it will be adopted the traditional approach used in the formulation of objective function, $P(x)$, of a flexible topology. It is defined by the ratio between the mutual strain energy and the compliance of the structure (Yin and Ananthasuresh 2001):

$$
P(x)=-\frac{U_{1}^{T} K U_{2}}{U_{3}^{\mathrm{T}} K_{3} U_{3}}
$$

where the numerator and the denominator represent the structural flexibility and stiffness measures respectively. In this formulation, the user does not need know the values of the weighting coefficients of the flexibility and stiffness of the structure. When the Eq. (8) is maximized, the mutual strain energy is maximized and the compliance is minimized simultaneously. The global stiffness matrix, $K_{3}$, in the denominator of Eq. (8) is obtained by restricting the degree of freedom of the point of application of the forces $F_{1}$ or $F_{2}$, shown the in Fig. 4. The displacement field, $U_{3}$, is derived from this condition.

Using the objective function model defined above, the topology optimization problem of the flexible stent cell will be:

Minimize:

$$
P(x)=-\frac{U_{1}^{T} K U_{2}}{U_{3}^{T} K_{3} U_{3}}
$$

Subjected to:

$$
\begin{aligned}
& f=\frac{V(x)}{V} \\
& K U_{1}=F_{1} \\
& K U_{2}=F_{2} \\
& K_{3} U_{3}=F_{3} \\
& 0 \leq x_{\text {min }} \leq x \leq 1
\end{aligned}
$$

From the stent design point of view, $F_{3}$ is the reaction force of the vessel wall on the stent cell due to the contact that occurs when the catheter bends. In fact, $F_{3}$ is equal to $F_{2}$ but in opposite direction. In this situation, the degrees of freedom of the point of application of $F_{1}$ are restricted for the computation of the $U_{3}$ from the Eq. (11).

\section{Algorithm for the Generation of the Optimal Topologies}

The stent volume fraction is the only active constraint of both formulations above described. Furthermore, the maximization of the hardening and flexibility has a large number of design variables. These design variables are the relative density of each finite element from design space. For problems of this nature, the optimality criteria method is the optimizer more efficient to obtain the optimal solution (Mayer et al., 1996; Maute et al., 1998; Sigmund, 2001; Yin and Ananthasuresh, 2001). Therefore, it will be used in this work to provide the stent cell optimal topologies.

The optimality criteria method is based on the Kuhn-Tucker conditions applied to the Lagrangian of the objective function and constraints. It can be demonstrated that the stationary condition of the Lagrangian of the elastic-plastic strain energy defined by the Eq. (1) subjected to the constraints (4), (5) and (6) of the hardening maximization problem of the stent cell is given by (Guimarães, 2005):

$$
\begin{gathered}
B_{w}=\frac{\left\{\varepsilon^{e}\right\}^{T} \frac{\partial\left[E_{p}\right]}{\partial x^{e}}\left\{\varepsilon^{e}\right\}}{\frac{\sum_{i=1}^{N}\left\{\varepsilon^{e}\right\}^{T} \frac{\partial\left[E_{p}\right]}{\partial x^{e}}\left\{\varepsilon^{e}\right\}}{N}} \\
\lambda \frac{\partial V\left(x^{e}\right)}{\partial x^{e}} \\
\left(x^{e}\right)^{j+1}=\left(B_{w}\right)^{\eta}\left(x^{e}\right)^{j}
\end{gathered}
$$

where $\lambda$ is the Lagrangian multiplier of the volume constraint, $N$ is the number of finite elements used in the discretization of the design space, $\eta$ is a numerical damping coefficient to decrease the convergence rate in the computation of the optimal topology, $j$ denotes the iteration index used in the updating of the topology and $i$ is an index which represents the summation of all finite elements from the design space. Equation (12) depends on the sensitivity of the plastic modulus, which is the derivative of the Eq. (3) with respect to the $x^{e}$.

In this work, it will be employed the SIMP (solid isotropic material with penalization) approach as an interpolation model of relative density of the stent cell. In this case, the optimal topology is totally defined and free of porous (Sigmund, 2001) and the sensitivity of the plastic modulus in the Eq. (12) is easily computed.

In the case of the flexibility maximization problem of the stent cell, the stationary condition of the Lagrangian applied to the Eq. (8), (4), (9), (10), (11) and (12) is defined by (Guimarães, 2005):

$$
\begin{aligned}
& A=\frac{1}{U_{3}^{T} K_{3} U_{3}} U_{2}^{T} \frac{\partial K}{\partial x^{e}} U_{1}- \\
& -\frac{\mathrm{U}_{1}^{\mathrm{T}} K U_{2}}{\left(U_{3}^{T} K_{3} U_{3}\right)^{2}} U_{3}^{T} \frac{\partial K_{3}}{\partial x^{e}} U_{3}+\lambda \frac{\partial V\left(x^{e}\right)}{\partial x^{e}} \\
& \left(x^{e}\right)^{j+1}=\left(\mathrm{x}^{\mathrm{e}}\right)^{j}-\mathrm{A}^{\mathrm{j}}
\end{aligned}
$$

where the relative density in the iteration $j+1$ was updated based on the gradient of the Lagrangian multiplied by -1 , which represents the steepest descent direction of this optimization problem. Once again, the SIMP model defined for the flexible stent cell will be used in the updating of the topology.

Unfortunately, Eq. (13) and Eq. (15) does not guarantee a stable convergence since abrupt changes may occur in the formation of the 
topology. In order to stabilize the formation of the topology, the following procedure was used in this work (Maute et al., 1998; Sigmund, 2001; Yin and Ananthasuresh, 2001):

$$
\max \left[0.001,\left(x^{e}\right)^{j}-\mathrm{m}\right] \leq\left(x^{e}\right)^{j+1} \leq \min \left[\mathrm{l},\left(x^{e}\right)^{j}+m\right]
$$

where $m$ is the move limit that represents a maximum permissible value of the change of relative density in each iteration. The role of this parameter is to avoid the formation of discontinuities in the topology during the optimization. The choice of the value of this parameter depends on the behavior of objective function to be optimized. In the present paper, the value of $m$ for each problem will be chosen observing the convergence process of the optimal topology. If the objective function begins to oscillate, the value of $m$ is reduced so that the convergence process became stable.

Figure 5 illustrates the steps of the topology optimization algorithm to be used in both problems, maximization of the flexibility and hardening. Although they are different problems treated separately, it will be used the same methodology in the derivation of the optimal topologies based on the optimality criteria method. Initially, all finite elements from design space have relative density equal to volume fraction chosen by the user. For the maximization problem of the flexibility, the nodal displacement fields $U_{1}, U_{2}$ and $U_{3}$ are obtained by solving the equilibrium equations (9), (10) and (11). These displacements will be extracted from a subroutine implemented in the $\mathrm{Matlab}^{\circledR}$ (Sigmund, 2001). For the hardening problem, it will be used the finite element software ANSYS ${ }^{\mathbb{Q}}$ in order to extract the elastic and plastic strain field from the stent design space. Subsequently, the objective function sensibility is calculated. Finally, the Lagrangian multiplier, $\lambda$, from volume constraint is determined using a bi-sectioning algorithm and the topology of stent cell is updated. The subroutines of updating of the stent cell topology were implemented in Matlab ${ }^{\mathbb{B}}$ (Sigmund, 2001).

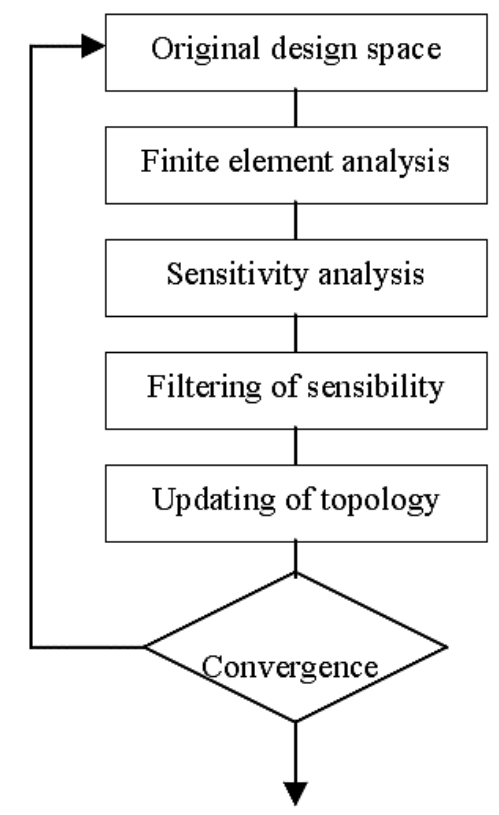

Figure 5. Flowchart of the stent cell optimization topology algorithm.

In topology optimization, there still exists a natural tendency to the formation of regions like checkerboards (Sigmund, 2001). In these regions, the optimal material distribution is not continuous and oscillates from solid to void. The more efficient approach for the treatment of this problem, to be used in this paper, is the relative density filtering of the finite elements of structure (Sigmund, 2001). In this approach, the relative density of each finite element will be computed according to the weighting average of the strain energy sensibility of the neighboring elements. Thus, before the updating of the topology using the Eq. (16), the sensitivity of the strain energy of each finite element is computed, as illustrated in the flowchart (Fig. 5). The objective of this procedure is to obtain a more uniform material distribution and without the checkerboard patterns.

\section{Structural Model for the Stent Cell Optimization}

Figure 6 shows the design space and the boundary conditions that simulate the balloon expansion for the hardening maximization problem (Ahmad and Barrett, 1999). Due to symmetry of the stent cell, it will only be considered the half of its design space. The traction displacements, $\Delta u$, of same magnitude applied to the upper and lower corners from the right edge simulate the stent expansion process caused by the balloon. Nonlinear finite element analysis from software ANSYS ${ }^{\circledR}$ simulates this expansion process several times during the optimization.

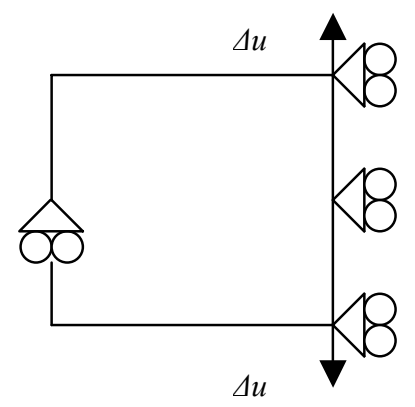

Figure 6. Structural model for the maximization of the hardening of the stent cell.

Table 1. Parameters of the stent cell structural model subjected to the plastic strain.

\begin{tabular}{|c|c|}
\hline Parameters of the stent model & Magnitude \\
\hline Young's modulus & $190 \times 10^{9} \mathrm{~N} / \mathrm{m}^{2}$ \\
\hline Tangent modulus & $1300 \times 10^{6} \mathrm{~N} / \mathrm{m}^{2}$ \\
\hline Yield stress & $250 \times 10^{6} \mathrm{~N} / \mathrm{m}^{2}$ \\
\hline Poisson's ratio & 0.3 \\
\hline Stent cell length & $1.5 \times 10^{-3} \mathrm{~m}$ \\
\hline Stent cell height & $1.25 \times 10^{-3} \mathrm{~m}$ \\
\hline Traction displacement & $0.22 \times 10^{-3} \mathrm{~m}$ and $0.44 \times 10^{-3} \mathrm{~m}$ \\
\hline
\end{tabular}

Table 1 shows the geometrical parameters as well as the material properties to be used in the hardening maximization of the stent cell (Norton, 1996; Serruys and Kutryk, 1998). In this situation, it will be generated two hardened stent cell topologies corresponding to two different values of $\Delta u$ described in the Tab. 1 . The smaller displacement, $\Delta u=0.22 \mathrm{~mm}$, models the expansion of a stent to be implanted in an artery with a small diameter. Similarly, the displacement, $\Delta u=0.44 \mathrm{~mm}$, simulates the expansion of the stent in a vessel with a larger diameter.

The vertical load $F_{1}$ simulates the force applied by the vessel wall on the stent when the catheter bends. The dummy load $F_{2}$ is the desirable direction of the stent flexibility. It is applied at the middle of the bottom edge of the design spaces shown in the Fig. 7 and Fig. 8. Moreover, the flexible stent cell should maintain its shape when 
subjected to reaction force applied by the blood vessel wall, $F_{3}$. Then, the stent cell stiffness should also be maximized due to the reaction force, $F_{3}$.
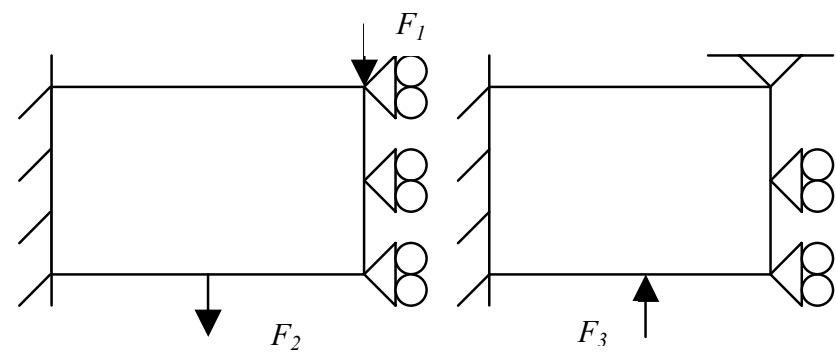

Figure 7. Structural model for the maximization of the flexibility of the stent cell with the fixed left edge.

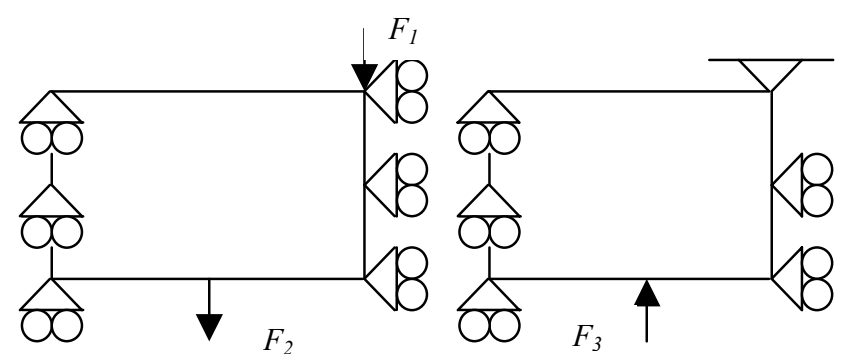

Figure 8. Structural model for the maximization of the flexibility of the stent cell with the left edge free to move in the horizontal direction.

Table 2. Parameters of the flexible stent cell structural model subjected to the large elastic strain.

\begin{tabular}{|c|c|}
\hline Parameters of the stent model & Magnitude \\
\hline Young's modulus & 1 \\
\hline Poisson's ratio & 0.3 \\
\hline Stent cell length & 1 \\
\hline Stent cell height & 0.83 \\
\hline$F_{1}$ & 1 \\
\hline$F_{2}$ & 1 \\
\hline$F_{3}$ & -1 \\
\hline
\end{tabular}

The physical models for the maximization of the flexibility of the stent cell are shown in the Fig. 7 and Fig. 8. The material properties, dimensions of the design space and the magnitude of the forces applied to both structural models are defined in the Tab. 2. In this situation, the parameters of the stent model were normalized. The difference between both models is the boundary condition applied in the left edge. The cantilever beam illustrated in the Fig. 7 do not take account the movements in the interface of the flexible longitudinal structure with the stent cell design space subjected to the plastic strain. On the other hand, in the model shown in the Fig. 8 , all nodes from the left edge are free to move in the horizontal direction. In practice, the interface between the hardened and flexible structures of the stent cell is free to move as in the vertical direction as in the horizontal one. Thus, the structural model shown in the Fig. 8 is more realistic when compared to the model from Fig.
7. However, both models will be used to provide the stent flexible topologies.

\section{Results and Discussion}

The deformed shape of the stent cell material distributions, with maximum hardening corresponding to the traction displacements of $0.22 \times 10^{-3} \mathrm{~m}$ and $0.44 \times 10^{-3} \mathrm{~m}$, are illustrated in the Fig. 9 and Fig. 10 , respectively. As the traction displacement of magnitude $\Delta u$ is applied on the upper and lower right corners from the design space (Fig. 6), the total displacement of stent cell in the vertical direction after the expansion is equal to $0.44 \mathrm{~mm}$ and $0.88 \mathrm{~mm}\left(0.22 \times 10^{-3} \mathrm{~m}\right.$ and $0.44 \times 10^{-3} \mathrm{~m}$ multiplied by two), respectively. Therefore, the percentual vertical displacements of the stent cell with respect to the design space height are $35 \%$ and $70 \%(0.44 \mathrm{~mm} / 1.25 \mathrm{~mm}$ and $0.88 \mathrm{~mm} / 1.25 \mathrm{~mm})$. For these situations, the stent diameter will also increase $35 \%$ and $70 \%$ since this is proportional to the circunferential length of the cross section.

The structures shown in the Fig. 9 and Fig. 10 were provided from the contours of the optimal topologies generated by using the algorithm shown in the Fig. 5 (Guimarães, 2005). In both cases, the topologies were computed from a mesh with $30 \times 25$ finite elements. Since each finite elements analysis used in simulation of expansion of the stent cell is nonlinear, it was necessary to divide the total solution interval in 200 steps in order to solve the structural equilibrium equations set during the updating of the topologies. This value is recommended by the program ANSYS $^{\circledR}$ to avoid the divergence of the nonlinear solution process (ANSYS Inc., 2002). Moreover, the program ANSYS ${ }^{\circledR}$ should select the best solver to be used in the solution of the nonlinear equilibrium equations.

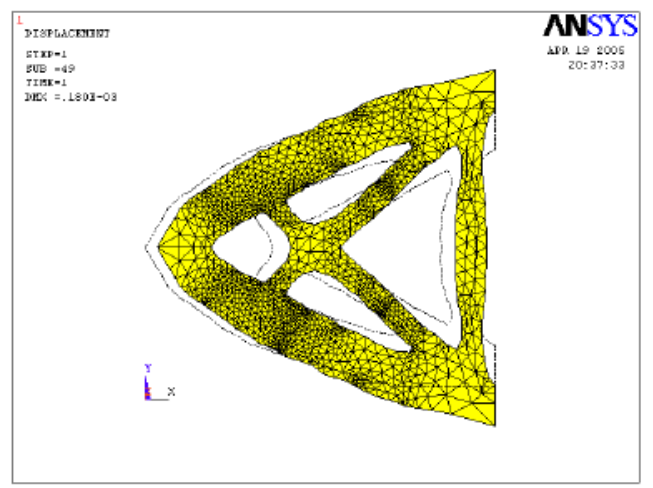

Figure 9. Stent cell optimal material distribution subjected to the expansion displacement of $0.22 \times 10-3 \mathrm{~m}$.

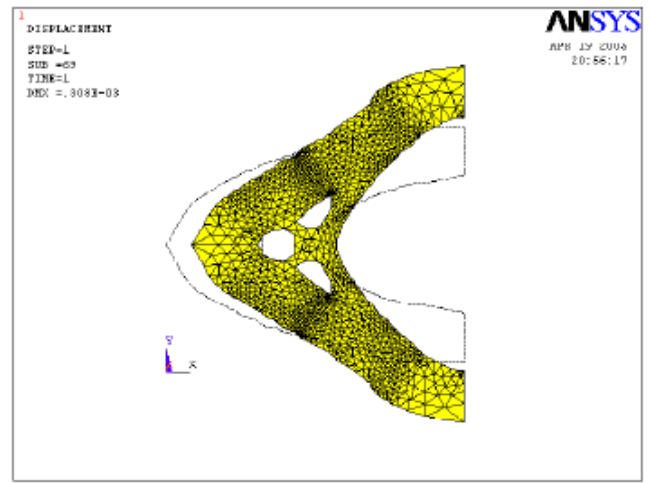

Figure 10. Stent cell optimal material distribution subjected to the expansion displacement of $0.44 \times 10-3 \mathrm{~m}$. 
There are significant differences between the stent cell material distributions shown in the Fig. 9 and 10. In the topology with maximum hardening subjected to the expansion displacement of $0.22 \times 10^{-3} \mathrm{~m}$ (Fig. 9), the material is distributed in the vertical direction by connecting the upper and lower corners of the right edge from the design space. When this stent cell expands, the plastic strain distribution concentrates in this region (Guimarães, 2005). For the stent cell topology illustrated in the Fig. 10, it is not more seen the presence of this material distribution close to right edge. In addition, it can be also observed that the details of this topology are shifted to the left from the design space when compared to material distribution shown in the Fig. 9. In this case, the plastic strain field concentrates in a region close to the 3 holes of the stent cell material distribution shown in the Fig. 10 (Guimarães, 2005).

Figures 11 and 12 illustrate the deformed shapes of the flexible stent cell topologies that were optimized from the structural models shown in the Fig. 7 and Fig. 8, respectively. In the reanalysis process, the force $F_{1}$ was replaced by a displacement of normalized magnitude equals to 0.083 applied on the flexible stent optimal topologies. For these situations, it can be observed the presence of a material sudden variation in some regions of the topologies illustrated in the Fig. 11 and 12. The major role of these discontinuous regions placed in the stent topologies is to absorb the elastic deflection energy and to improve their flexibility.

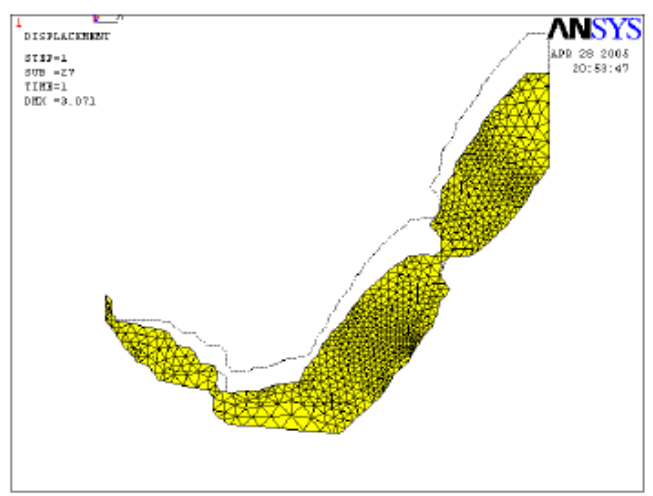

Figure 11. Flexible stent cell optimal material distribution with the left edge fixed.

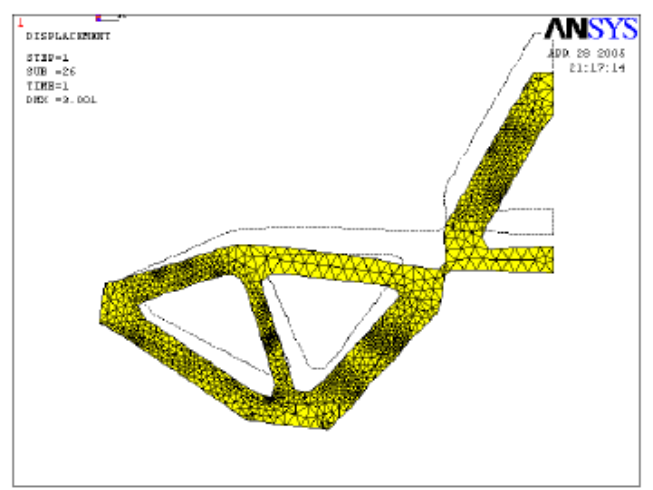

Figure 12. Flexible stent cell optimal material distribution with the left edge free to move in the horizontal direction.

The topologies with maximum hardening shown in this paper have some differences when compared with the geometry of the commercial stents illustrated in the Fig. 2. The stent cell material distribution subjected to the expansion of $0.22 \times 10^{-3} \mathrm{~m}$ (Fig. 9) is similar to the topology of the compliance minimization problem (Sigmund, 2001). When the expansion displacement was increased to $0.44 \times 10^{-3} \mathrm{~m}$, the relative density of the local material distribution in vertical direction close to the right edge was decreased. From this observation, it can be concluded that the stent cell topology subjected to the expansion of $0.44 \times 10^{-3} \mathrm{~m}$ is less stiff and more easily expanded when compared to the other example. Another difference is the size of the holes near the middle of the left edge in the two topologies. These details suggest that the stent cell design is based on the diameter of the artery where the stent will be implanted. On the other hand, in commercial stents, the cell geometry does not depend on the diameter of the blood vessel where the stent will be implanted.

The flexible stent cell topologies provided in this paper have two features that are not found in commercial stents. The sudden variation of material distribution of the flexible topologies shown in this paper is not usually found in commercial stents. As shown in the Fig. 2, commercial stents use continuous structural elements of curved geometry in the longitudinal direction in order to improve the flexibility of the cell. Moreover, commercial stents are designed only to absorb the elastic strain energy during the implant procedure. In the stent cells topologies illustrated in this work, the stiffness of point of application of the forces on the design space was also maximized. In commercial stents, only the material stiffness guarantees their structural integrity in large deflections.

The figure 2 illustrates the plane model of the stent after the union of the flexible and stiff topologies shown in the Fig. 10 and Fig. 11. Subsequently, this plane model was wrapped into a cylindrical shape to generate the three-dimensional stent model. The diameter of this stent model before the angioplasty is $3 \mathrm{~mm}$ (Guimarães et al., 2006). If the plane model shown in the Fig. 2 is subjected to the percentage displacement equals to $70 \%$, the diameter of three-dimensional stent will increase $2.1 \mathrm{~mm}$. Thus, the final diameter of the expanded stent will be $5.1 \mathrm{~mm}$. In a typical angioplasty, this stent model could be used, for example, in a diseased vessel diameter into a range of $4 \mathrm{~mm}$ to $6 \mathrm{~mm}$.

\section{Conclusions}

In the present paper, it was proposed a methodology for the optimal topology design of the plane model of stainless steel stent cells by considering separately their flexibility and hardening. The stent cell topologies were computed using an algorithm based on the heuristics and in the optimality criteria method.

It was demonstrated that is possible to design stents cell using the topology optimization technique. Although there are significant differences between the topologies provided in the present work and in the commercial stents geometry, all examples met the flexibility and hardening criteria. The stent cells subjected to the plastic strain provided in this paper shown that the material distribution depends on the size of the artery diameter where they will be implanted. In commercial stents, the elastic deflection energy tends to distribute along the flexible structural element with curved geometry. In the flexible optimal topologies shown in the present paper, the elastic energy is concentrated in the regions with sudden variation of material distribution. Furthermore, the structural integrity of the flexible optimal topologies is preserved since the stiffness of the point of contact between the stent cell and the vessel wall is maximized.

\section{Acknowledgment}

The authors would like to acknowledge the financial support provided by the CNPq. 


\section{References}

Ahmad, A. and Barrett, P., 1999, Private Communication.

ANSYS User's Manual, 2002, ANSYS, Inc., P.O. Box 65, Houston, PA $15342-0065$.

Borgersen, S. and Sadeghi, R., 2000, Private Communication.

Duerig, T. W., Tolomeo, D. E., Wholey, M., 2000, “An Overview of Superelastic Stent Design", Proceedings of Shape Memory and Superelastic Technologies, Vol.9 (3/4), pp. 235-246.

Guimarães, T. A., 2005, "Application of the Topological Optimization Technique to the Stents Cells Design for Angioplasty" (In Portuguese), Ph.D. Thesis, Federal University of Uberlândia, Uberlândia, M.G., Brazil, $125 \mathrm{p}$.

Guimarães, T. A., Araujo, R., Langoni, A. M., Oliveira, S. A. G., 2006, "Simulation of the Expansion Process of Stents for Angioplasty by Hidroforming", $16^{\circ}$ Simposia in Mechanical Engineering, Federal University of Uberlandia, Uberlandia, M.G., Brazil.

Karasudhi, P., 1991, "Foundations of Solid Mechanics", Ed. Kluwer Academic Publishers, 439 p.

Maute, K., Schwarz, S., Ramm, E., 1998, "Adaptive Topology Optimization of Elastoplastic Structures", Structural Optimization , Vol.15, pp. 81-91.

Mayer, R. R., Kikuchi, N., Scott, R. A., 1996, "Application of Topological Optimization Techniques to Structural Crashworthiness",
International Journal for Numerical Methods in Engineering, Vol.39, pp. 1383-1403.

McClean, D. R. and Eigler, N. L., 2002, "Stent Design: Implications for Restenosis", Reviews in Cardiovascular Medicine, Vol.3, pp. 16-22.

Nishiwaki, S., Min, S., Yoo, J., Kikuchi, N., 2001, “Optimal Structural Design Considering Flexibility", Computer Methods in Applied Mechanics and Engineering, Vol.190, pp. 4457-4504.

Norton, R. L., 1996, "Machine Design: An Integrated Approach", Ed. Prentice Hall, 1048 p.

Rozvany, G. I. N., 1997, "Topology Optimization in Structural Mechanics", Ed. Springer Verlag, 376 p.

Serruys, P. W. and Kutryk, N. J. B., 1998, "Handbook of Stents Coronary", Ed. Martin Dunitz, 271 p.

Sigmund, O., 2001, “A 99 Line Topology Optimization Code Written in Matlab”, Structural Optimization, Vol.16, pp. 120-127.

Vanderplaats, G. N., 1999, "Numerical Optimization Techniques for Engineering Design", $3^{\text {rd }}$ edition, Vanderplaats Research \& Development Inc., Colorado Springs, 333 p.

Yin, L. and Ananthasuresh, G. K., 2001, "Topology Optimization of Compliant Mechanisms with Multiple Materials Using a Peak Function Material Interpolation Scheme", Structural Optimization, Vol. 23, pp.49-62. 